Научная статья

УДк 531.19

DOI https://doi.org/10.24866/VVSU/2073-3984/2021-3/158-168

C.В. Сёмкин ${ }^{1}$

В.П. Смагин ${ }^{2}$

Владивостокский государственный университет экономики и сервиса

Владивосток. Россия

\title{
Термодинамические функции и фрустрационные свойства магнетиков
}

В настоящей работе получены выражения, позволяющие вычислить свободную энергию и энтропию для магнитной системы, для которой есть точное или приближенное выражение для намагниченности. Вывод этих выражений опирается только на асимптотическое поведение намагниченности при увеличении внешнего поля и на значение гамильтониана магнитной системы в состоянии насыщения. Поэтому полученный результат легко может быть распространен на достаточно широкий класс магнитных моделей как чистых, так и с немагнитным разбавлением. Полученное выражение для магнитной энтропии может служить средством анализа фрустрированного состояния в системе. В качестве признака фрустрированности магнитной системы в работе используется отличие от нуля энтропии основного состояния. Примером магнитной системы, допускающей возникновение фрустраций, служит одномерная модель Изинга во внешнем поле с взаимодействием только ближайших соседей. Анализ выражения для намагниченности этой модели позволяет построить фазовую диаграмму основного состояния. Фазовыми переменными на этой диаграмме являются константа обменного взаимодействия ближайших соседей и величина внешнего поля. Диаграмма состоит из трех двумерных областей с положительной, отрицательной и нулевой намагниченностью соответственно. Границы между этими областями можно рассматривать как отдельные зоны диаграммы со своими значениями намагниченности. Анализ выражения для энтропии показывает, что энтропия основного состояния обращается в ноль во внутренних точках областей диаграммы, что говорит об отсутствии фрустраций в этих областях. Энтропия

${ }^{1}$ Сёмкин Сергей Викторович - канд. физ.-мат. наук, доцент кафедры информационных технологий и систем; e-mail: Li15@rambler.ru

${ }^{2}$ Смагин Виктор Павлович - д-р физ.-мат. наук, зав. лабораторией фундаментальной и прикладной физики; e-mail: Li15@rambler.ru

158 
основного состояния не равна нулю на границах областей с нулевой и ненулевой намагниченностью.

Ключевые слова и словосочетания: фрустрация, модель Изинга, фазовая диаграмма.

\author{
S.V. Semkin \\ V.P. Smagin \\ Vladivostok State University of Economics and Service \\ Vladivostok. Russia
}

\title{
Thermodynamic functions and frustration properties of magnets
}

In the present work, expressions are obtained that make it possible to calculate the free energy and entropy for a magnetic system, for which there is an exact or approximate expression for the magnetization. The derivation of these expressions is based only on the asymptotic behavior of the magnetization with an increase in the external field and on the value of the Hamiltonian of the magnetic system in the saturation state. Therefore, the result obtained can easily be extended to a fairly wide class of magnetic models, both pure and with nonmagnetic dilution. The obtained expression for the magnetic entropy can serve as a means of analyzing the frustrated state in the system. As a sign of frustration of the magnetic system, the work uses the difference from zero of the entropy of the ground state. The one-dimensional Ising model in an external field with the interaction of only the nearest neighbors is used as an example of a magnetic system that allows the occurrence of frustrations. Analysis of the expression for the magnetization of this model makes it possible to construct a phase diagram of the ground state. The phase variables in this diagram are the constant of the exchange interaction of the nearest neighbors and the magnitude of the external field. The diagram consists of three two-dimensional regions with positive, negative and zero magnetization, respectively. The boundaries between these areas can be considered as separate areas of the diagram with their own values of magnetization. Analysis of the expression for the entropy shows that the entropy of the ground state vanishes at the interior points of the areas of the diagram, which indicates the absence of frustrations in these areas. The entropy of the ground state is nonzero at the boundaries of regions with zero and nonzero magnetization.

Keywords: phase transitions, frustration, Ising model, phase diagram.

\section{Введение}

Классическая теория фазовых переходов в магнитных системах развивается уже достаточно давно и в настоящее время приобрела законченный вид [1; 2]. Однако существует целый ряд магнитных явлений, не укладывающихся в эту классическую теорию. К ним относятся разного рода неупорядоченные, разбавленные и аморфные магнетики [3; 4], а также так называемые фрустрированные магнетики. Явление магнитных фрустраций, обнаруженное в 70-х годах XX века, объясняется сильным вырождением основного состояния системы и невоз- 
можностью полного магнитного упорядочения даже при нулевой температуре [5]. И хотя по фрустрированным магнитным системам накоплен богатый экспериментальный материал и написано немало теоретических работ, не все экспериментальные факты и новые эффекты удалось объяснить, а ряд свойств фрустрированных систем еще недостаточно понятен [6].

При отсутствии фрустрации для любой термодинамической системы выполняется теорема Нернста (третье начало термодинамики), заключающаяся в том, что энтропия $S$ любой системы стремится к нулю, если к нулю стремится ее абсолютная температура $T$. Поэтому признаком фрустрации в системе можно считать отличие от нуля энтропии основного состояния [6]. Это дает теоретический инструмент для исследования фрустрированных систем, а именно: для определения, есть или нет фрустрация, нужно рассчитать энтропию системы и найти ее предел при $T \rightarrow 0$.

Для магнитных систем одной из основных характеристик является намагниченность $M$. Многими приближенными, а иногда и точными методами можно вычислить $M$ как функцию температуры $T$ и внешнего магнитного поля $H$ [7-9]. Поэтому в настоящей работе мы находим энтропию магнитной системы, основываясь на выражении для намагниченности $M=M(H, T)$ этой системы. Полученное выражение можно использовать для определения условий возникновения фрустраций. В качестве простого примера мы находим энтропию одномерной цепочки изинговских спинов с взаимодействием ближайших соседей и определяем условия, при которых в этой системе возникает фрустрация.

\section{Свободная энергия и энтропия магнетика}

Полная свободная энергия термодинамической системы [2]:

$$
F=-k T \operatorname{Ln} Z,
$$

где $k$ - постоянная Больцмана, $T$ - абсолютная температура, $Z$ - статистическая сумма системы, определяемая по формуле:

$$
Z=\sum \exp \left(-\frac{H}{k T}\right)
$$

Суммирование в (2) проводится по всем возможным состояниям системы, от которых зависит ее гамильтониан $H$. Во многих случаях гамильтониан магнитных систем имеет вид:

$$
H=H_{6}\left(m_{i}\right)-H \sum_{i} m_{i}
$$

где $m_{i}$ - нормированный на максимальное значение магнитный момент $i$-го магнитного атома, $H_{b}\left(m_{i}\right)$ - гамильтониан обменного взаимодействия, $H$ - внешнее магнитное поле. Из формул $(1-3)$ следует, что средний магнитный момент одного атома (намагниченность) вычисляется так:

$$
M=-\frac{\partial f}{\partial H},
$$


$f=\frac{F}{N}$ - удельная (на один атом) свободная энергия.

Вводя переменные $h=\frac{H}{k T}$ и $\varphi=\frac{f}{k T}$, запишем (4) в виде:

$$
M(h, T)=-\frac{\partial \varphi(h, T)}{\partial h} .
$$

Вычислим из (5) свободную энергию с помощью рассуждений, аналогичных [10]. Мы полагаем, что, если величина внешнего поля $H$ достаточно велика, наибольший вклад в (2) вносит слагаемое, соответствующее состоянию, в котором все магнитные моменты параллельны внешнему полю. Поэтому при больших $h$ :

$$
\varphi \approx \frac{\varepsilon_{p}}{k T}-h,
$$

где $\varepsilon_{p}$ - удельная энергия обменных взаимодействий в состоянии, когда все спины параллельны. Кроме того, намагниченность $M(h, T)$ при любом $T$ стремится к единице при $h \rightarrow \infty$. Используя это обстоятельство и асимптотическое равенство (6), получим:

$$
\varphi(h, T)=\frac{\varepsilon_{p}}{k T}-h+\int_{h}^{\infty}(M(\tilde{h}, T)-1) d \tilde{h} .
$$

Удельная энтропия $s$ связана с удельной свободной энергией соотношением [2]:

$$
s=-\frac{\partial f}{\partial T} .
$$

Используя это соотношение и равенство (7), получим:

$$
\frac{s}{k}=-\frac{\partial}{\partial T}\left(T \int_{h}^{\infty}(M(\tilde{h}, T)-1) d \tilde{h}\right),
$$

что можно переписать следующим образом:

$$
\frac{s}{k}=(1-M(h, T)) h+\int_{h}^{\infty}\left(1-M(\tilde{h}, T)+\frac{\partial M(\tilde{h}, T)}{\partial T}\right) d \tilde{h} .
$$

Формулы (7-9) можно использовать для вычисления свободной энергии и энтропии достаточно широкого класса моделей магнетиков. В частности, они применимы к моделям Изинга и Гейзенберга как для чистых, так и для разбавленных магнетиков (в последнем случае намагниченность кроме внешнего поля и температуры будет зависеть еще и от концентрации магнитных атомов [9]). В настоящей работе мы ограничимся рассмотрением одномерной цепочки изинговских спинов с взаимодействием только ближайших соседей. 


\section{Одномерная модель Изинга}

Одномерная модель Изинга была сформулирована и решена Эрнстом Изингом еще в 1925 году [10], представляет из себя цепочку спиновых переменных $\sigma_{i}$, пронумерованных по порядку индексом $i=1, \ldots N$, каждая из которых может принимать только два значения +1 и -1 . Гамильтониан этой модели имеет вид:

$$
H(\sigma)=-J \sum_{i=1}^{N} \sigma_{i} \sigma_{i+1}-H \sum_{i=1}^{N} \sigma_{i} .
$$

Если константа обменного взаимодействия $J>0$, модель описывает «ферромагнитное» взаимодействие спинов - энергия взаимодействия минимальна в случае, когда спины параллельны. Если же $J<0$, модель описывает «антиферромагнитное» взаимодействие - энергия минимальна, когда знаки спинов чередуются.

Намагниченность в расчете на один узел при $N \rightarrow \infty$ для одномерной модели Изинга равна:

$$
M(h, K)=\frac{s h(h)}{\sqrt{\operatorname{sh}^{2}(h)+e^{-4 K}}},
$$

где $K=\frac{J}{k T}$.

Получить этот результат можно различными способами: вычислив собственные значения трансфер-матрицы [10], составив рекуррентное выражение [10] или рассмотрев ренормгрупповое преобразование конечного масштаба [9].

При малых значениях $T$ выражение для намагниченности (11) приближенно равно:

$$
M(H, T) \approx \frac{\operatorname{sign}(H)}{\sqrt{1+4 \exp \left(-\frac{2(2 J+|H|)}{k T}\right)}} .
$$

Из этого выражения видно, что намагниченность в основном состоянии $(T \rightarrow 0)$ определяется знаком внешнего магнитного поля $H$ и знаком выражения. Если $2 J+|H|>0$ и $\operatorname{sign}(H)=+1$, то намагниченность в основном состоянии равна +1 , а если при этом $\operatorname{sign}(H)=-1$, то -1 . Эту ситуацию можно изобразить на фазовой диаграмме (рис. 1) в координатах $H$ и $J$. Область положительной намагниченности лежит в правой части диаграммы между прямыми $H=0$ и $J=-\frac{H}{2}$, а область отрицательной - симметрично относительно оси $J$. На границе этих областей $(H=0)$ намагниченность в основном состоянии равна нулю. Если же $2 J+|H|<0$, то, как видно из (12), намагниченность в основном состоянии равна нулю независимо от знака внешнего поля. Эта область находится в нижней 
части диаграммы (см. рисунок), ниже линии. На самой этой линии намагниченность равна $-\frac{1}{\sqrt{5}}$ при $H<0$ и $\frac{1}{\sqrt{5}}$ при $H>0$.

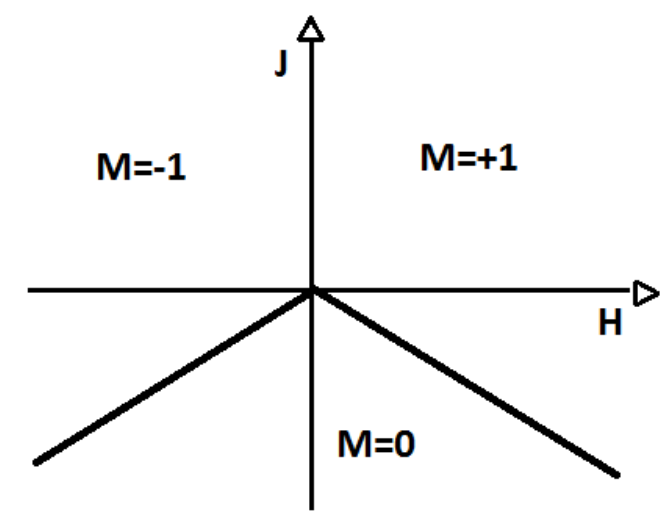

Рис. Диаграмма основного состояния одномерного изинговского магнетика во внешнем поле

Можно предположить, что во всех внутренних точках диаграммы основное состояние не вырождено и фрустрации в этих областях отсутствуют. Если это предположение верно, фрустрации в системе можно ожидать только на границах областей. Для того чтобы показать, что это действительно так, найдем энтропию основного состояния одномерного изинговского магнетика. Подставляя (11) в (7), найдем удельную свободную энергию одномерной модели Изинга:

$$
f(h, K)=-J-k T \operatorname{Ln}\left(\sqrt{\left.{s h^{2}(h)+e^{-4 K}+\operatorname{ch}(h)}\right)} .\right.
$$

Удельную энтропию модели найдем либо из формулы (9), либо продифференцировав (13) по $T$ :

$$
\begin{aligned}
& \frac{s(h, K)}{k}=-\frac{2 K e^{-4 K}}{1-e^{-4 K}}\left(1-\frac{c h(h)}{\sqrt{s^{2}(h)+e^{-4 K}}}\right)+\operatorname{Ln}\left(\sqrt{s h^{2}(h)+e^{-4 K}+c h(h)}\right)- \\
& -\frac{h s h(h)}{\sqrt{s h^{2}(h)+e^{-4 K}}} .
\end{aligned}
$$

При отсутствии внешнего поля $(h=0)$ энтропия рассчитывается следующим образом:

$$
\frac{s(0, K)}{k}=\operatorname{Ln}\left(1+e^{-2 K}\right)+\frac{2 K e^{-2 K}}{1+e^{-2 K}} .
$$

Рассматривая пределы этого выражения при $K \rightarrow+\infty$ и при $K \rightarrow-\infty$ (то есть в случае и ферромагнитного, и антиферромагнитного взаимодействия при $T \rightarrow 0)$, легко показать, что они оба равны нулю. Другими словами, при $H=0$ 
и при всех $J \neq 0$ в системе отсутствует фрустрация. Если же $J=0$, то, как видно из (15), энтропия системы при любых значениях температуры, в том числе и при $T \rightarrow 0$, равна $k \operatorname{Ln} 2$. Иначе говоря, парамагнетик можно рассматривать как некоторый тривиальный пример фрустрированной магнитной системы. Выражение (14) не определено при $J=0$, но если в выражении для свободной энергии (13) с самого начала взять $J=0$, то получим энтропию парамагнетика как функцию внешнего магнитного поля:

$$
s_{0}(H)=k L n\left(2 c h\left(\frac{H}{k T}\right)\right)-\frac{H}{T} \operatorname{th}\left(\frac{H}{k T}\right),
$$

откуда видно, что энтропия основного состояния обращается в ноль при $H \neq 0$.

Рассмотрим теперь энтропию основного состояния одномерной модели Изинга при $J \neq 0$ во внешнем магнитном поле. При $J>0$ и $T \rightarrow 0$ первое слагаемое в правой части равенства (14) и сумма второго и третьего, как легко показать, стремятся к нулю, что означает отсутствие фрустраций во всей ферромагнитной $(J>0)$ области фазовой диаграммы (см. рисунок). Если же $J<0$, то из (14) следует, что $2 J+|H| \neq 0$ энтропия основного состояния равна нулю, а при $2 J+|H|=0$, то есть на границах областей с намагниченностью \pm 1 и 0 (см. рисунок), равна $k \operatorname{Ln} \frac{1+\sqrt{5}}{2}$. Иначе говоря, на всей линии $2 J+|H|=0$ в системе присутствует фрустрация. Этот результат согласуется с полученным в работе [6].

\section{Заключение}

В работе получено выражение для свободной энергии и энтропии магнитной системы, основанное на использовании намагниченности как функции температуры и внешнего поля. Это выражение можно применять к любой модели магнетика, для которой точным или приближенным методом находится намагниченность. В качестве примера рассмотрена одномерная модель Изинга с взаимодействием ближайших соседей. Построена магнитная фазовая диаграмма модели, а также проведен анализ конфигурационных особенностей основного состояния и фрустрационных свойств системы.

1. Ма Ш. Современная теория критических явлений. - Москва: Мир, 1980. - 296 с.

2. Квасников И. А. Термодинамика и статистическая физика: в 2 т. Т. 2: Теория равновесных систем. - Москва: Едиториал УРСС, 2002. - 432 с.

3. Изюмов Ю.А., Медведев М.В. Теория магнитоупорядоченных кристаллов с примесями. - Москва: Наука, 1970. - 271 с.

4. Доценко В.С. Критические явления в спиновых системах с беспорядком // Успехи физических наук. - 1995. - Т. 165 (5). - С. 481-528.

5. Vannimenus J., Toulouse G. Theory of the frustration effect. II. Ising spins on a square lattice // Journal of Physics C: Solid State Physics. - 1977. - № 10. - P. 537.

6. Фрустрационные свойства одномерной модели Изинга / А. В. Зарубин, Ф. А. КассанОглы, А. И. Прошкин, А. Е. Шестаков // Журнал экспериментальной и теоретической физики. - 2019. - Т. 155, Вып. 5. - С. 914-946. 
7. Смагин В.П., Сёмкин С. В. Рекуррентные решетки и самосогласованные уравнения в модели Изинга // Территория новых возможностей. Вестник Владивостокского государственного университета экономики и сервиса - 2019. - № 2. - С. 139-149.

8. Сёмкин С. В., Смагин В. П., Гусев Е.Г. Модель Изинга с немагнитным разбавлением на рекурсивных решетках // Теоретическая и математическая физика. - 2020. - Т. 202, №. 2. - С. 304-311.

9. Сёмкин С. В., Смагин В. П. Приближенные методы в теории чистых и разбавленных магнетиков: монография. - Владивосток: Изд-во ВГУЭС, 2019. - 220 с.

10. Бэкстер Р. Точно решаемые модели в статистической механике.- Москва: Мир, 1985. $-488 \mathrm{c}$.

\section{References}

1. Ma Sh. Sovremennaya teoriya kriticheskih yavlenij. - Moskva: Mir, 1980. - 296 s.

2. Kvasnikov I. A. Termodinamika i statisticheskaya fizika: v 2 t. T. 2: Teoriya ravno-vesnyh sistem. - Moskva: Editorial URSS, 2002. - 432 s.

3. Izyumov Yu. A., Medvedev M. V. Teoriya magnitouporyadochennyh kristallov s primesyami. - Moskva: Nauka, 1970. - 271 s.

4. Docenko V.S. Kriticheskie yavleniya v spinovyh sistemah s besporyadkom // Uspekhi fizicheskih nauk. - 1995. - T. 165 (5). - S. 481-528.

5. Frustracionnye svojstva odnomernoj modeli Izinga / A. V. Zarubin, F. A. Kassan-Ogly, A. I. Proshkin, A. E. Shestakov // Zhurnal eksperimental'noj i teoreticheskoj fiziki. 2019. - T. 155, Vyp. 5. - S. 914-946.

6. Smagin V.P., Semkin S. V. Rekurrentnye reshetki i samosoglasovannye uravneniya v modeli Izinga // Territoriya novyh vozmozhnostej. Vestnik VGUES. - 2019. - № 2. S. 139-149.

7. Semkin S. V., Smagin V. P., Gusev E. G. Model' Izinga s nemagnitnym razbavleniem na rekursivnyh reshetkah // Teoreticheskaya i matematicheskaya fizika. - 2020. - T. 202, №. 2. - S. 304-311.

8. Semkin S. V., Smagin V. P. Priblizhennye metody v teorii chistyh i razbavlennyh magnetikov: monografiya. - Vladivostok: Izd-vo VGUES, 2019. - 220 s.

9. Bekster R. Tochno reshaemye modeli v statisticheskoj mekhanike. - Moskva: Mir, 1985. $488 \mathrm{~s}$.

(C) С.В. Сёмкин, 2021

(C) В.П. Смагин, 2021

Для цитирования: Сёмкин С.В., Смагин В.П. Термодинамические функции и фрустрационные свойства магнетиков // Территория новых возможностей. Вестник Владивостокского государственного университета экономики и сервиса. - 2021. - Т. 13, № 3. - С. 158165.

For citation: Semkin S. V., Smagin V.P. Thermodynamic functions and frustration properties of magnets, The Territory of New Opportunities. The Herald of Vladivostok State University of Economics and Service, 2021, Vol. 13, № 3, pp. 158-165.

DOI https://doi.org/10.24866/VVSU/2073-3984/2021-3/158-165

Дата поступления: 13.08.2021. 